\section{Farmacoepidemiologia no Brasil: evolução e perspectivas}

\author{
Pharmacoepidemiology in Brazil: \\ evolution and prospects
}

Lia Lusitana Cardozo de Castro 1

1 Centro de Informação de Medicamentos,

Universidade de Mato Grosso, Rua Lago Êrie 455, apto. 11, 79070-340 Campo Grande, MS, Brasil
Abstract This work recovers the historical development of pharmacoepidemiology in Brazil. The role played by the following events is particularly dealt with the influence of the pharmacological education programs coordinated by professor Carlos Lacaz, author of the first Brazilian textbook on iatrogenic diseases; the clinical pharmacy program offered by the Pan-American Health Organization, which provided a number of Brazilian professionals their first contact with the Program of Drug Surveillance by Intensive Hospital Monitoring; the first doctoral on drugs presented at São Paulo University; the attempt of the Division of Medicine of the Brazilian Health Ministry to include Pharmacovigilance among its more important aims. Also the founding of the Brazilian Association for Drug Surveillance are reported. Emphasis is also placed on the implementation of several Drug Information Centers in Brazil, and the new thrust thus provided to Drug Epidemiology. Finally, conclusions are drawn on the conditions for gathering efforts already available in universities and health service agencies nationwide in order to implement pharmacoepidemiological research in Brazil.

Key words Epidemiology; Pharmacoepidemiology; Drug Surveillance; Drug Rational Use; Public Health
Resumo O presente trabalho apresenta o desenvolvimento da farmacoepidemiologia no Brasil. Enfatiza o papel dos programas de educação continuada em farmacologia coordenados pelo professor Carlos Lacaz, autor do primeiro livro brasileiro sobre doenças iatrogênicas. Ressalta o papel do programa de formação em farmácia clínica da Organização Pan-Americana de Saúde, onde vários profissionais brasileiros tiveram o primeiro contato com o Programa de farmacovigilância por Monitorização Intensiva Hospitalar. Refere também às primeiras teses de doutorado sobre medicamentos defendidas na Universidade de São Paulo. Relata a tentativa da Divisão de Medicamentos do Ministério da Saúde do Brasil de se direcionar para a farmacovigilância. Tece considerações sobre a I Oficina de Trabalho sobre medicamentos organizada pelas Secretarias Municipal e Estadual de Saúde de São Paulo. Enfatiza a criação da Sociedade Brasileira de Vigilância de Medicamentos, e sua importância como estimuladora da farmacoepidemiologia. Analisa o impulso do setor dinamizado pela implantação dos Centros de Informações de Medicamentos, no país. Conclui que já existem condições para se incrementar a pesquisa farmacoepidemiológica no Brasil.

Palavras-chave Epidemiologia; Farmacoepidemiologia; Farmacovigilância; Uso Racional de Medicamentos; Saúde Pública 


\section{Introdução}

O Brasil, como a maioria dos países do mundo, foi atingido pela tragédia da talidomida. Atualmente existem cerca de 7 mil brasileiros vitimados pelo uso desse medicamento na década de 60. No entanto, a percepção do risco relacionado ao uso de medicamentos vem se desenvolvendo lentamente no país (Anônimo, 1996).

Podem ser considerados como pioneiros em alertar os profissionais de saúde para o risco relacionado ao uso de medicamentos, Lacaz, Colbert e Teixeira, que não só publicaram no Brasil o primeiro livro sobre o assunto intitulado doenças iatrogênicas, como promoveram, na primeira metade da década de 70 , cursos de extensão em farmacoterapia, que até hoje seriam bastante atuais pelo enfoque prioritário que imprimiam ao uso racional de medicamentos Lacaz et al., (1970).

Outra importante influência para essa percepção foi o programa de formação de farmacêuticos clínicos patrocinado pela Organização Pan-Americana de Saúde (OPAS) no Hospital Aguirre da Universidade do Chile, Santiago, onde alguns profissionais farmacêuticos tiveram contato com um programa de vigilância hospitalar intensiva nos moldes do Boston Colaborative Drug Surveillance Castro, (1996).

Em conseqüência desse contato inicial, professores chilenos e espanhóis ligados à área estiveram no Brasil e, sob essa influência, foram desenvolvidos alguns trabalhos hospitalares enfocando reações adversas a medicamentos. Surgem também, na segunda metade da década de 70 , e na primeira década de 80 , algumas teses de doutorado, na Universidade de São Paulo, usando o método epidemiológico para estudar problemas relacionados a medicamentos. Destacamos alguns exemplos desses trabalhos, tais como Aspectos Epidemiológicos do Consumo de Medicamentos Psicotrópicos pela População de Adultos do Distrito de São Paulo (Tancredi, 1979), Contribuição para o Estudo da Eficácia e Toxicidade de Algumas Associações Medicamentosas no Tratamento da Tuberculose Pulmonar (Castro, 1981), e Saúde e Classe Social: Um Estudo sobre Morbidade e Consumo de Medicamentos Barros, (1983).

Na segunda metade da década de 80 , mais precisamente no período compreendido entre os anos de 1985 e 1987, a Divisão de Me- dicina do Ministério da Saúde do Brasil (Dimed) tenta implementar uma nova política de vigilância sanitária de medicamentos e faz contato com o professor Juan Ramon Laporte, e mais tarde com o professor Gianni Tognoni. Este contato não frutificou no problemático espaço da administração pública brasileira e migra para a Escola de Saúde Pública, no Rio de Janeiro. O resultado é bem representado pela tradução do livro Epidemiologia do Medicamento, de Laporte e Tognoni, que até hoje é a única coletânea de textos em português sobre o assunto. Nessa época, há uma tomada de consciência que a prática fiscalizadora da vigilância sanitária de medicamentos é insuficiente para levar ao uso adequado dos mesmos pela população, e se inicia um direcionamento rumo à farmacoepidemiologia Rosenfeld, (1989).

No ano de 1989, com o objetivo de chamar a atenção para a necessidade de garantir o uso racional de medicamentos nos serviços de saúde e incentivar a formação de profissionais em farmacoepidemiologia, foi organizada a primeira oficina de trabalho nesse campo do conhecimento, em São Paulo. Esta primeira oficina de trabalho contou com a orientação do professor Gianni Tognoni e a participação de 81 profissionais médicos, enfermeiros e farmacêuticos pertencentes às administrações regionais das Secretarias de Saúde Estadual e Municipal de São Paulo. Desse encontro surgiram importantes protocolos de pesquisa Informe sobre a Oficina, (1989).

Um acontecimento relevante para o desenvolvimento da farmacoepidemiologia no Brasil foi a criação, em São Paulo, da Sociedade Brasileira de Vigilância de Medicamentos (Sobravime) em 30 de novembro de 1990 (Sobravime, 1991). A Sobravime é uma sociedade civil sem fins lucrativos, constituída por pessoas físicas e jurídicas com interesse em estudos e investigações científicas sobre vigilância de insumos farmacêuticos e medicamentosos. Desde sua criação, essa entidade vem incentivando estudos e investigações científicas sobre diferentes aspectos do medicamento Sobravime, (1991).

A Sobravime realizou quatro congressos, onde foram apresentados inúmeros trabalhos (sob a forma de posters, cursos, conferências, mesas-redondas, etc.) enfocando diferentes aspectos da questão dos medicamentos. A tendência desses trabalhos, ao longo dos anos, tem-se mostrado ascendente em relação tan- 
to à quantidade como à qualidade das pesquisas apresentadas Sobravime, (1997).

Simultaneamente à criação da Sobravime, surge o Grupo de Prevenção ao Uso Indevido de Medicamentos (GPUIM), que realizou o Seminário Abortamento provocado em Fortaleza, riscos e responsabilidades, com ampla repercussão na imprensa. Desde então, esse grupo vem atuando na área de medicamentos em atividades de pesquisa e extensão voltadas para o uso racional de medicamentos, representando significativo elemento para o desenvolvimento da farmacoepidemiologia no país.

Nos anos 90 são implantados no Brasil vários Centros de Informação de Medicamentos (CIM), que por suas características de trabalho permitem documentar como a população e os profissionais estão utilizando os medicamentos e a eventual ocorrência de reações adversas aos mesmos. Estes dados constituem elementos importantes para a farmacovigilância e para os estudos de utilização de medicamentos, ambos objeto da farmacoepidemiologia. Acrescente-se que a reunião de profissionais com conhecimentos complementares - médicos, epidemiologistas, estatísticos, entre outros - necessários aos CIM, contribuem significativamente para a emergência de grupos interessados em realizar estudos farmacoepidemiológicos Castro et al., (1997). O fato desses centros estarem localizados em várias regiões do país é especialmente propício para a criação de um Sistema Nacional de Farmacovigilância (Sobravime, 1996).

Em reunião específica de Centros de Informação de Medicamentos realizada no IV Congresso da Sociedade Brasileira de Vigilância de Medicamentos, foi discutida a interação entre CIM e centros de farmacovigilância, sendo apresentada a experiência do Centro de Informação de Medicamentos da Universidade Federal de Mato Grosso do Sul (CIM/UFMS), onde foi iniciado um Programa de Monitorização de Reações Adversas em Menores de 12 Anos Castro \& Vicente, (1997).

O Sistema de Farmacovigilância no Brasil vem sendo cogitado desde a Constituição de 1988, e é referido na Lei Orgânica de Saúde Brasil, (1991), porém começa realmente a ser discutido após a reunião que ocorreu em Buenos Aires, Argentina, intitulada I Reunião para Elaboração de Estratégias para Implementação de Sistemas de Farmacovigilância na América Latina, com o apoio da OPAS/OMS. Após essa reunião, foi nomeada uma comissão que elaborou uma proposta inicial para a implantação de um plano piloto de farmacovigilância no país, o que ainda não ocorreu Arraes, (1996).

Em novembro de 1996, realizou-se em Fortaleza o I Seminário Brasileiro de Farmacoepidemiologia, promovido pelo GPUIM na Universidade Federal do Ceará (UFCE). Nesse Seminário, ocorreu também outro importante fato que foi a assinatura de convênio entre a UFCe, através do GPUIM, e a Secretaria de Estado de Saúde do Ceará, para iniciar o Programa de Farmacovigilância naquele Estado (GPUIM, 1996). Os dados iniciais do Programa de Farmacovigilância do Ceará foram apresentados ao IV Congresso da Sociedade Brasileira de Vigilância de Medicamentos Souza et al., (1997). Acredita-se que esta experiência venha a se reproduzir em outros estados que já dispõem de pessoal qualificado para isto, como é o caso de Mato Grosso do Sul, contribuindo, desta forma, para a estruturação de um Sistema Nacional de Farmacovigilância no Brasil (Castro, 1997).

A OPAS promoveu, de 23 a 25 de abril de 1997, sob a coordenação da Administración Nacional de Medicamentos, Alimentos y Tecnología Médica (ANMAT) - Ministerio de Salud y Acción Social de la Nación Argentina, uma reunião intitulada Guia para el Estabelecimiento y Funcionamiento de 'Centros de Farmacovigilância' en América Latina. Nessa reunião, participaram alguns especialistas brasileiros (Argentina, 1997), e esse encontro contribuiu para a aceleração do processo de implantação da farmacovigilância no Brasil. Estimulado pelos resultados dessa reunião, o CIM/UFMS, através de dois de seus pesquisadores, inicia um Programa de Sensibilização de Médicos Pediatras para a Notificação de Reações Adversas a Medicamentos e Imunobiológicos em Menores de 12 Anos (Vicente, 1997).

A Secretaria Nacional de Vigilância Sanitária do Ministério da Saúde promoveu, em julho de 1997, uma reunião técnica para a constituição do Sistema Nacional de Farmacovigilância. Os participantes dessa reunião discutiram a proposta que já vem sendo trabalhada desde 1995, e elaboraram um projeto de organização e implantação do Sistema Nacional de farmacovigilância Brasil, (1997).

A produção científica em Farmacoepidemiologia no Brasil, em recente trabalho relaciona 101 pesquisas, diversificadas. O temas dessas pesquisas representam, principalmente, o interesse pessoal dos pesquisadores por 
determinados assuntos (Castro \& Simões, 1995). No entanto, cresce em todo o país o interesse pela farmacoepidemiologia. Em 1993 foram formados no Centro-Oeste dois grupos de pesquisadores em farmacoepidemiologia, atuando na UFMS, Campo Grande, Mato Grosso do Sul, e na Universidade Federal de Mato Grosso (UFMT), Cuiabá. O grupo de pesquisadores em Uso Racional de Medicamentos de Mato Grosso do Sul (Grupuram) coordena o CIM/UFMS, ministra a disciplina de farmacoepidemiologia no curso de Mestrado em Saúde Coletiva da mesma universidade. Já foram produzidos pelos pesquisadores desse grupo estudos importantes para o Estado de Mato Grosso do Sul (Castro, 1997; Rossini \& Castro, 1996; Vicente \& Castro, 1997; Oshiro \& Castro, 1997; Toledo et al., 1996).

Foram defendidas algumas dissertações de Mestrado elaboradas por alunos-pesquisadores do grupo e orientadas por docentes pertencentes ao mesmo grupo, tendo como tema questões relevantes relacionadas ao uso de medicamentos no Estado de Mato Grosso do Sul (Almeida, 1997; Carneiro, 1997; Ishikawa, 1997). Estão em andamento mais quatro dissertações que utilizam metodologias inovadoras de intervenção comunitária, visando ao uso racional de medicamentos (Ajalla, 1997; Silva, 1997; Vicente, 1997; Oshiro, 1998).

O grupo de pesquisadores em farmacoepidemiologia da UFMT participa do programa de pós-graduação em Saúde e Ambiente dessa Universidade, na sub-área de Farmacovigilância, com duas linhas de pesquisa: Abuso de Drogas e Consumo de Medicamentos, nas quais se encontram quatro trabalhos em andamento (Vanzeler \& Martins, 1998). Na sub-área de farmacovigilância do programa de saúde e ambiente foram defendidas duas dissertações de mestrado abordando o uso de drogas lícitas e ilícitas (Souza, 1996; Rodrigues, 1998).

Em 1994, na Universidade Estadual de Campinas (Unicamp), estrutura-se mais um produtivo grupo de pesquisa, predominantemente voltado para o uso de medicamentos em hospitais (Mendes, 1998). Esse grupo participa do programa de pós-graduação da Unicamp com quatro dissertações defendidas (Pfafenbach, 1997; Braga, 1997; Fonseca, 1998; Leite, 1998) e um estudo comunitário em desenvolvimento (Fonseca, 1998).

Em 1995, a Universidade do Estado de São Paulo (Unesp) introduz no currículo do Curso de Especialização em Saúde Pública a dis- ciplina de farmacoepidemiologia, e passa a oferecê-la regularmente (Unesp, 1995). A partir dessa data, o grupo formado na Unesp vem intensificando pesquisas que já estavam sendo desenvolvidas isoladamente por alguns professores. Atualmente esse grupo dirige seus trabalhos para desenvolver indicadores de medida de qualidade de uso de medicamentos no Brasil (Simões \& Fegadolli, 1996; Motta \& Simões, 1997). Além dos grupos anteriormente citados, muitos pesquisadores desenvolvem trabalhos tendo como objeto a epidemiologia de medicamentos. São bastante divulgados os trabalhos produzidos na Universidade Federal de Minas Gerais na Universidade Federal do Rio de Janeiro, e na Escola Nacional de Saúde Pública .

A década de 90 tem se caracterizado pelo crescimento do conhecimento acumulado em farmacoepidemiologia não só no Brasil como em toda América Latina. Nesse crescimento, a ANMAT/Argentina tem desempenhado um importante papel ao promover reuniões em todos os países da região, visando implementar a farmacovigilância no continente sul-americano.

Estimulada por essas reuniões, a Secretaria Nacional de Vigilância Sanitária promoveu alguns encontros com especialistas de várias regiões do país, com o objetivo de estruturar o Sistema Nacional de Farmacovigilância, cuja implantação espera-se que ocorra em breve. Grupos de pesquisadores interessados em estudos epidemiológicos sobre medicamentos vêm promovendo eventos de caráter nacional, como, por exemplo, o I Seminário Brasileiro de Farmacoepidemiologia, organizado pelo GPUIM em 1996. Esses esforços, somados ao trabalho pioneiro da Sobravime, também de âmbito nacional, vêm proporcionando a integração dos profissionais interessados em farmacoepidemiologia no país.

Por outro lado, as tentativas relativamente bem-sucedidas de grupos nacionais que atuam em pesquisa e docência em farmacoepidemiologia, aliadas à perspectiva de cooperação internacional com grupos com tradição na área, como a Universidade Autônoma de Barcelona, Espanha, a McGill de Montreal, Canadá, o Instituto Mário Negri de Milão, Itália, e outros grupos que também se iniciam na área, como a Universidade Nova de Lisboa, Portugal, permitem pensar que num futuro muito próximo superaremos a fase dos estudos pré-farmacoepidemiológicos de caráter pontual tornando- 
se possível o desenvolvimento de trabalhos multicêntricos de interesse nacional.

$\mathrm{O}$ atual estágio da pesquisa e da docência em farmacoepidemiologia no país permitenos afirmar que em breve essa área do conhecimento se consolidará no Brasil, e trazendo ações fundamentais de Saúde Pública, como a implantação do Sistema Nacional de Farmacovigilância e a difusão da terapêutica informada, contribuindo de forma importante para a concretização de uma prática imprescindível para a saúde e para a qualidade de vida da população o uso racional e seguro de medicamentos.

\section{Referências}

Ajalla ME 1997. A Prática Farmacêutica nos Tratamentos de DSTs em Campo Grande, Mato Grosso do Sul. Dissertação de Mestrado (em andamento). Universidade Federal de Mato Grosso do Sul, Campo Grande.

Almeida MG 1997. Reações Adversas às Vacinas do PNI no Período de 1990 a 1996 em Campo Grande, Mato Grosso do Sul. Dissertação de Mestrado (em andamento). Universidade Federal de Mato Grosso do Sul, Campo Grande.

Anônimo 1996. Talidomida: o inferno ainda arde. Pharmácia Brasileira Ano 1, jan.-fev.

Arraes PS 1996. Farmacovigilância: até que enfim no Brasil! Saúde em Debate 40-50.

Barros MBA 1983. Saúde e Classe Social: um Estudo sobre Morbidade e Consumo de Medicamentos no Tratamento da Tuberculose Pulmonar. Tese de Doutorado. Faculdade de Medicina, USP, Ribeirão Preto.

Braga T 1997. Prevalência de Prescrição de Medicamentos para Pacientes Internados em um Hospital-Escola, Idosos versus Não-Idosos. Dissertação de Mestrado. Universidade Estadual Paulista, Campinas.

Brasil 1991. Ministério da Saúde. Assessoria de Comunicação Social. Lei Orgânica de Saúde. 2. ${ }^{\mathrm{a}}$ ed. Assessoria de Comunicação Social, Brasília, D.F.

Brasil 1997. Ministério da Saúde. Secretaria de Vigilância Sanitária. Reunião sobre o Sistema Nacional de Farmacovigilância. Relatório, Brasília, D.F.

Carneiro CAN 1997. Contribuição ao Uso Racional de Medicamentos nos Hospitais de Campo Grande, Mato Grosso do Sul: Estudo Relativo aos Conhecimentos sobre Medicamentos de Farmacêuticos, Médicos, Enfermeiros e Administradores. Dissertação de Mestrado (em andamento). Universidade Federal de Mato Grosso do Sul, Campo Grande.

Castro LLC 1981. Contribuição ao Estudo da Eficácia e Toxicidade de Algumas Associações Medicamentosas no Tratamento da Tuberculose Pulmonar. Tese de Doutorado. Faculdade de Saúde Pública, Universidade de São Paulo, São Paulo.

Castro LLC 1996a. Farmacoepidemiologia no Brasil, uma História Recente. Conferência. I Seminário de Farmacoepidemiologia. Livro de Resumos, Fortaleza.

Castro LLC 1996b. Situação Atual da Farmacoepidemiologia no Brasil. II Conferência de Farmacoepidemiologia. Resumo de Comunicações, Associação Nacional de Farmácias, Lisboa.
Castro LLC 1997a. Estudo de Algumas Características do Automedicação em Campo Grande, Mato Grosso do Sul. Relatório de Pesquisa CNPq. Universidade Federal de Mato Grosso do Sul, Campo Grande, mimeo.

Castro LLC 1997b. Situação Atual da Farmacovigilância em Mato Grosso do Sul/ Brasil. Administración Nacional de Medicamentos, Alimentos y Tecnología Médica/ ANMAT. Reunião para La Implementación del Guía para el Estabelecimiento y Funcionamiento de Centros de Farmacovigilância en América Latina. Buenos Aires, mimeo.

Castro LLC, Simões MJ 1995. Análise Descritiva dos Estudos sobre Impacto dos Medicamentos em Populações no Brasil no Período de 1990 a 1994. Anais do Seminário Internacional de Farmacêuticos, São Paulo.

Castro LLC, Vicente MG 1997. Interação entre Centros de Informação de Medicamentos e Centros de Monitorização de Reações Adversas. Conferência. IV Congresso Brasileiro de Vigilância de Medicamentos, Curitiba, mimeo.

Castro LLC et al. 1997. Serviços de informação de medicamentos: estratégias para o desenvolvimento da farmacoepidemiologia no país. In JRA Bonfim \& VL Mercucci (orgs.) - A Construção da Política de Medicamentos. Hucitec/Sobravime, São Paulo.

Fonseca E 1998. O Padrão de Prescrição para os Idosos atendidos no SUS de Piracicaba. Dissertação de Mestrado (em andamento). Universidade Estadual de Campinas, Campinas.

Fonseca M 1998. Uso de Medicamentos na Gravidez, uma Abordagem Farmacoepidemiológica. Dissertação de Mestrado. Universidade Estadual Paulista, Campinas.

GPUIM 1996. I Seminário Brasileiro de Farmacoepidemiologia. Livro de Resumos. Departamento de Farmácia, Fortaleza.

Informe sobre a Oficina 1989. Oficina de Trabalho sobre Epidemiologia de Medicamentos em Desenvolvimento nos Serviços Públicos de Saúde de São Paulo. São Paulo, mimeo.

Ishikawa AAG 1997. Intoxicação Aguda Voluntária por Medicamentos em Campo Grande, Mato Grosso do Sul. Dissertação de Mestrado (em andamento). Universidade Federal de Mato Grosso do Sul, Campo Grande.

Lacaz et al. 1970. Doenças Iatrogênicas. Sarvier, São Paulo. 
Leite DP 1998. Padrão de Prescrição para Pacientes Pediátricos Hospitalizados: uma Abordagem Farmacoepidemiológica. Dissertação de Mestrado. Universidade Estadual de Campinas, Campinas.

Mendes GBB 1998. Informação sobre Atividades de Pesquisa em Farmacoepidemiologia Desenvolvida na Unicamp, Campinas, fax.

Ministerio da Salud y Acción Social de la Nación Argentina 1997. Administración Nacional de Medicamentos, Alimentos y Tecnología Médica. Guía para el Estabelecimiento y Funcionamiento de Centros de Farmacovigilância en América Latina, Buenos Aires, folder.

Motta MA, Simões MJS 1997. Estudo do Uso de Medicamentos em Unidades de Saúde em Ribeirão Preto, São Paulo. Anais do IV Congresso Brasileiro de Vigilância de Medicamentos, Curitiba.

Oshiro ML 1998. Avaliação do Potencial de uma Intervenção para a Promoção do Uso da Terapia de Reidratação Oral (TRO) através da Educação dos Vendedores de Medicamentos nas Farmácias. Dissertação de Mestrado (em andamento). Universidade Federal de Mato Grosso do Sul, Campo Grande.

Oshiro ML, Castro LLC 1997. Conhecimento do Grau de Informação das Mães de Crianças de 0 a 4 Anos, sobre Terapia de Reidratação Oral (TRO). IV Congresso Brasileiro de Vigilância de Medicamentos. Programação, Resumos dos Currículos dos Palestrantes e dos Trabalhos Apresentados, Curitiba.

Pfafenbach G 1997. Estudo de Utilização de Medicamentos em Contexto Hospitalar, Exemplo dos Benzodiazepínicos. Dissertação de Mestrado. Universidade de Farmacoepidemiologia, Universidade Estadual de Campinas, Campinas.

Rodrigues MS 1996. Utilização e Armazenamento de Medicamentos e Produtos Naturais para Fins Terapêuticos em Cuiabá, Mato Grosso. Dissertação de Mestrado. Universidade Federal de Mato Grosso, Cuiabá.

Rosenfeld S 1989. O uso de medicamentos no Brasil. In J-R Laporte, G Tognini \& S Rosenfeld. Epidemiologia de Medicamentos, Hucitec/Abrasco, São Paulo, Rio de Janeiro.

Rossini A, Castro LLC 1996. Behavior in Guided Selfmedication in the City of Campo Grande, Mato Grosso do Sul State, Brazil. Pharmacy World \& Science 18(5): A20.
Silva MA 1997. O Verso e o Reverso do Medicamento: uma Questão de Educação em Saúde? Dissertação de Mestrado (em andamento). Universidade Federal de Mato Grosso do Sul, Campo Grande.

Simões MJS,\& Fegadolli C 1996. Consumo de Medicamentos por Prescrição Médica na Assistência Básica à Saúde no Município de Araraquara. I Seminário Brasileiro de Farmacoepidemiologia. Livro de Resumos, Fortaleza.

Sobravime 1991. Estatutos da Sociedade Brasileira de Vigilância de Medicamentos. Boletim n. ${ }^{\circ}$ 1, dez.-jan. Sobravime 1996. Dossiê Centros de Informações de Medicamentos. Boletim n. ${ }^{\circ} 20$.

Sobravime 1997. IV Congresso Brasileiro de Vigilância de Medicamentos. Programação, Resumo dos Trabalhos, Currículos dos Palestrantes e dos Trabalhos Apresentados, Curitiba.

Souza DP 1996. O Perfil Epidemiológico do Uso de Drogas entre Estudantes do $1 .^{\circ}$ e 2..$^{\circ}$ Graus da Rede Estadual de Ensino no Espaço Socialmente Organizado de Cuiabá 1996. Dissertação de Mestrado, Universidade Federal de Mato Grosso, Cuiabá.

Souza NR et al. 1997. Sistema de Notificação Voluntária de Reações Adversas a Medicamentos no Ceará. IV Congresso Brasileiro de Vigilância de Medicamentos. Programação, Resumo dos Trabalhos, Currículo dos Palestrantes e dos Trabalhos Apresentados, Curitiba.

Tancredi FB 1979. Aspectos Epidemiológicos de Consumo de Medicamentos pela População de Adultos do Distrito de São Paulo. Tese de Doutorado. Faculdade de Saúde Pública. Universidade de São Paulo, São Paulo.

Toledo I, Bof DL, Castro LLC 1996. Assessment of antibacterical drug consumption in a university hospital. Pharmacy World \& Science 18(5): A21.

Unesp - Universidade Estadual Paulista 1997. Curso de Especialização em Saúde Pública. Faculdade de Ciências Farmacêuticas, Araraquara, folder.

Vanzeler MLA, Martins DTO 1998. Grupo de Farmacovigilância da Universidade Federal de Mato Grosso, Cuiabá.

Vicente MG 1997. Estudo de uma Metodologia Adequada para Sensibilização a Notificação de Reações Adversas em Menores de 12 Anos: o Caso dos Pediatras de Mato Grosso do Sul. Dissertação de Mestrado (em andamento). Universidade Federal de Mato Grosso do Sul, Campo Grande. 